\title{
Visualization of structures and cosmic flows in the local Universe
}

\author{
Daniel Pomarède, ${ }^{1}$ Hélène Courtois, ${ }^{2,3}$ and R. Brent Tully ${ }^{3}$ \\ ${ }^{1}$ CEA/Saclay - Irfu, 91191 Gif-sur-Yvette Cedex, France \\ ${ }^{2}$ University of Lyon; UCB Lyon 1/CNRS/IN2P3; IPN Lyon, France \\ ${ }^{3}$ Institute for Astronomy (IFA), University of Hawaii, 2680 Woodlawn Drive, HI 96822, USA
}

\begin{abstract}
A visualization of 3D structures and cosmic flows is presented using information from the Extragalactic Distance Database V8k redshift catalog and peculiar velocities from the Cosmicflows- 1 survey. Structures within a volume bounded at $8000 \mathrm{~km} \mathrm{~s}^{-1}$ on the cardinal Supergalactic axes are explored in terms of both displaying the positions of the 30,124 galaxies of the catalog and its reconstructed luminosity density field, corrected to account for growing incompleteness with increasing distance. Cosmography of the local Universe is discussed with the intent to identify the most prominent structures, including voids, galaxy clusters, filaments, and walls. The mapping also benefits from precise distance measures provided through the Cosmicflows- 1 observational program. Three-dimensional visualizations of the coherent flows of galaxies in the nearby Universe are presented, using recent results based on reconstruction of cosmic flows with the Wiener filter approach. The three major components of the Milky Way's motion, namely expulsion from the Local Void, infall toward the Virgo Cluster, and the bulk flow of the historic Local Supercluster toward the Great Attractor are illustrated using different visualization techniques and analyzed in light of the cosmography derived from the V8k redshift and Cosmicflows-1 distance catalogs.
\end{abstract}

Keywords. atlases, galaxies: distances and redshifts, large-scale structures of universe

\section{Introduction}

Visualization of 3D structures and cosmic flows is a critical ingredient of research into the cosmography of the local Universe. The purpose of cosmography is to characterize the morphology of features pertaining to the hierarchy of cosmological structures, such as voids, groups, clouds, sheets, clusters, filaments, chains, superclusters, and walls. Since structures at all scales are not static in the Hubble flow, cosmography also has to deal with kinematic information. Maps that assume relative distances based on galaxy redshifts are distorted from true 3D positions. The analysis of cosmic flows in the context of the spatial distribution of charted structures involves identification of attractors and other sources of motions. In turn, the reconstruction of flows provides new insights into their source-density field. In this context, accurate determinations of distances and the peculiar velocity of galaxies are required.

\section{The Cosmicflows Project}

The objective of the Cosmicflows Project is to improve our knowledge of the cosmography of the nearby Universe, with emphasis on the identification of attractors. A key issue is to measure the radial component of the galaxies' deviant motion from the Hubble cosmic expansion. The radial peculiar velocity, $v_{\mathrm{pec}}$ is expressed as a function of the velocity relative to the cosmic microwave background (CMB), the distance $(d)$, and the value of the Hubble constant $\left(\mathrm{H}_{0}\right)$, i.e., $v_{\text {pec }}=v_{\mathrm{CMB}}-d \mathrm{H}_{0}$. Within $10 \mathrm{Mpc}$, the 
Cepheid period-luminosity relation, the tip-of-the-red-giant-branch method, and the surface brightness fluctuation luminosity indicators provide measurements of distances with $10 \%$ accuracy. Farther, up to $200 \mathrm{Mpc}$, the galaxy neutral Hi gas luminosity-rotation rate (Tully-Fisher) correlation gives distance measurements with an accuracy of $\sim 20 \%$. Hi observations have most recently been conducted using the NRAO $100 \mathrm{~m}$ (diameter) Robert C. Byrd Green Bank Telescope, complemented in the southern sky with observations at the Parkes telescope. The photometry is acquired with several systems including the University of Hawaii's $2.2 \mathrm{~m}$ telescope. A first release of measurements of distances and peculiar velocities of 1797 galaxies was contained in the CosmicFLows-1 Catalog presented in Tully et al. (2008). A 3D velocity and density reconstruction of these peculiar velocities using the Wiener filter technique is presented in Courtois et al. (2012).

\section{The Extragalactic Distance Database Catalogs}

Our study of the cosmography of the local Universe benefits from the catalogs made available through the Extragalactic Distance Database presented in Tully et al. (2009). In addition to the CosmicFlows-1 Catalog, the V8k Catalog consists of 30,124 galaxies with redshifts within $8000 \mathrm{~km} \mathrm{~s}^{-1}$ along each axis of the supergalactic coordinate system defined by de Vaucouleurs et al. (1991). To account for the distortions caused by the influence of the Virgo Cluster, redshift positions within $3000 \mathrm{~km} \mathrm{~s}^{-1}$ are adjusted following a numerical action-flow model. Redshift distortions associated with virial velocities within clusters are also corrected for.

A weakness in the use of these catalogs is found in their growing incompleteness with increasing distance. A description that takes this bias into account is offered by the reconstruction of the luminosity-density field obtained by smoothing the distribution of galaxies corrected with a Schechter function, as described in H. Courtois et al. (in prep.).

\section{Cosmographical maps of the local Universe}

Cosmography calls upon the tools of visualization. An assembly of individual galaxies forms a cloud of points, each one possibly attached to a pointing velocity vector. Reconstructed density fields are described on uniform grids, visualized with several techniques such as isosurface reconstruction. Velocity fields are visualized using vectors or streamlines. To address these needs, the SDVISION interactive visualization software is designed to explore structures and cosmic flows in three dimensions, and produce maps and exploratory movies, as illustrated in Fig. 1.

One of the most striking features in the structure of the local Universe is the dense concentration of galaxies identified by de Vaucouleurs (1956) that in large measure defines the equator of the Supergalactic coordinate system. Galaxies in this plane, at $S G Z=0$, are displayed in Fig. 2 (left). The Milky Way, located at the center, belongs to the Local Supercluster, whose main component is the Virgo Cluster. A dense filament connects Virgo to the region of the Centaurus Cluster, as can be further appreciated in Fig. 2 (right). At a distance similar to the Virgo Cluster but at negative $S G Y$ lies the Fornax Cluster. At $7000 \mathrm{~km} \mathrm{~s}^{-1}$ along the positive $S G Y$ axis lies the Great Wall, which includes the Coma Cluster. At intermediate distances on the scale considered here, we find three major concentrations labelled Pavo-Indus, Perseus-Pisces, and the Southern Wall. In terms of cosmography, another striking feature is the presence of voids with dimensions as large as $5000 \mathrm{~km} \mathrm{~s}^{-1}$. These two maps are affected by the growing incompleteness with increasing distance. This is corrected for in the 3D map shown in Fig. 1, which illustrates the preponderance of the Great Wall as the most important structure in the local Universe, and the relatively low importance of our Local Supercluster. Visualizations of 


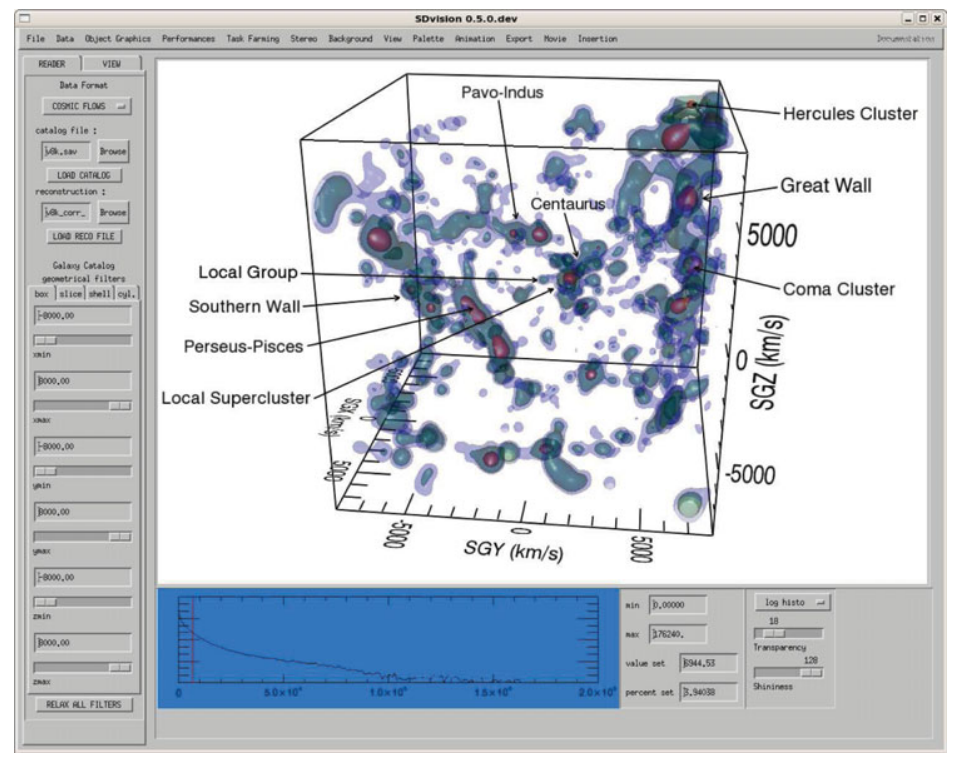

Figure 1. Visualization of the V8k Catalog smoothed density field with SDvision using multiple semi-transparent isosurfaces and providing some elements of cosmography.
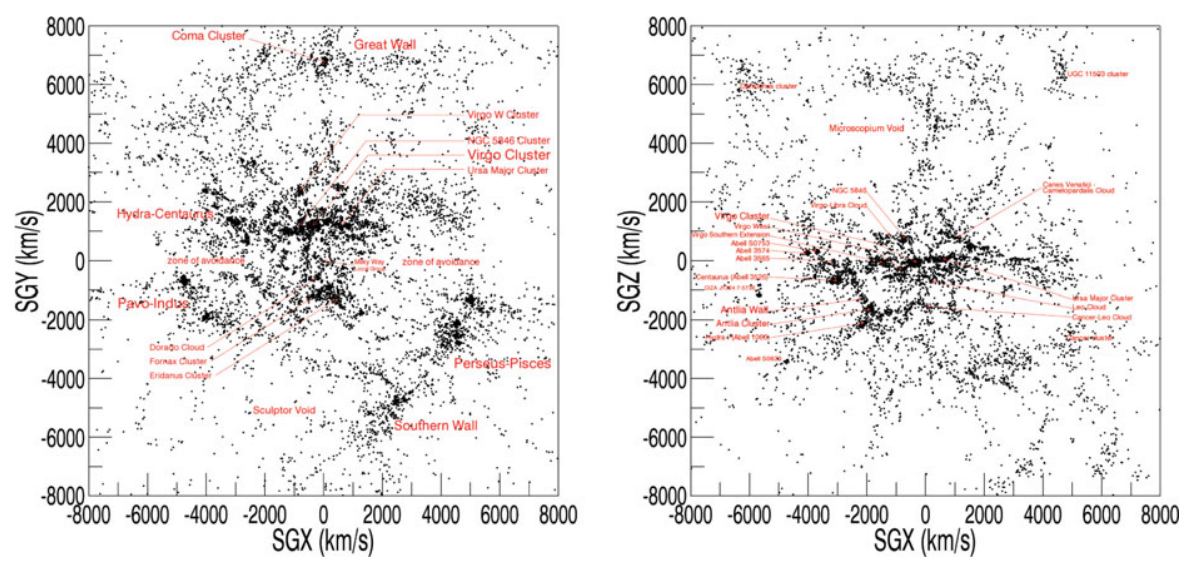

Figure 2. Slices of the local Universe. These two projections display the distributions of galaxies for $-1000 \leqslant S G Z\left(\mathrm{~km} \mathrm{~s}^{-1}\right) \leqslant 1000$ (left) and $500 \leqslant S G Y\left(\mathrm{~km} \mathrm{~s}^{-1}\right) \leqslant 2500$ (right).

the reconstructed velocity field in the nearby Universe are presented in Fig. 3, together with a high-density isosurface reconstructed from the V8k catalog. From these maps, we can infer the presence of a major attractor in the region of the Centaurus Cluster. Cosmic flows visualized as streamlines seeded in the $S G X=0$ plane are displayed in Fig. 4. This map also shows the underlying density field colored from black (underdense) to red (overdense), the V8k galaxies, and the Cosmicflows-1 galaxies with radial peculiar velocities attached (blue and red arrows for inward and outward moving galaxies, respectively). This map reveals the presence of the very underdense 'Local Void' in the immediate vicinity of the Milky Way. This void is seen in both the distribution of galaxies and the reconstructed source field. The map illustrates the clearing of the voids in the form of outbound flows, as already reported by Tully et al. (2008). The map also reveals that the Milky Way is caught in a flow toward the Virgo Cluster, associated mostly with 
Virgo's gravitational infall. Other attractors and convergent flows seen in this map are discussed in H. Courtois et al. (in prep.).
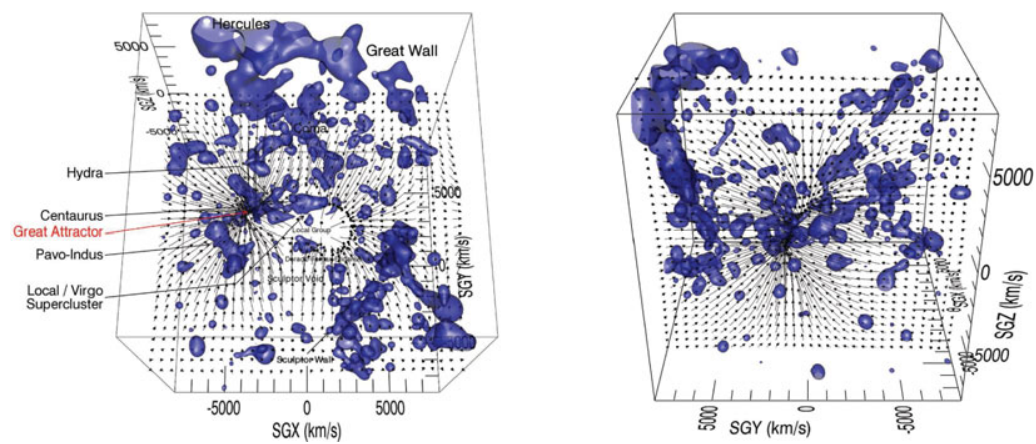

Figure 3. Visualization of the Cosmicflows-1 reconstructed velocity field in the Supergalactic Plane, $S G Z=0$ (left), and in the $S G Y-S G Z$ plane going through the Centaurus Cluster (right).

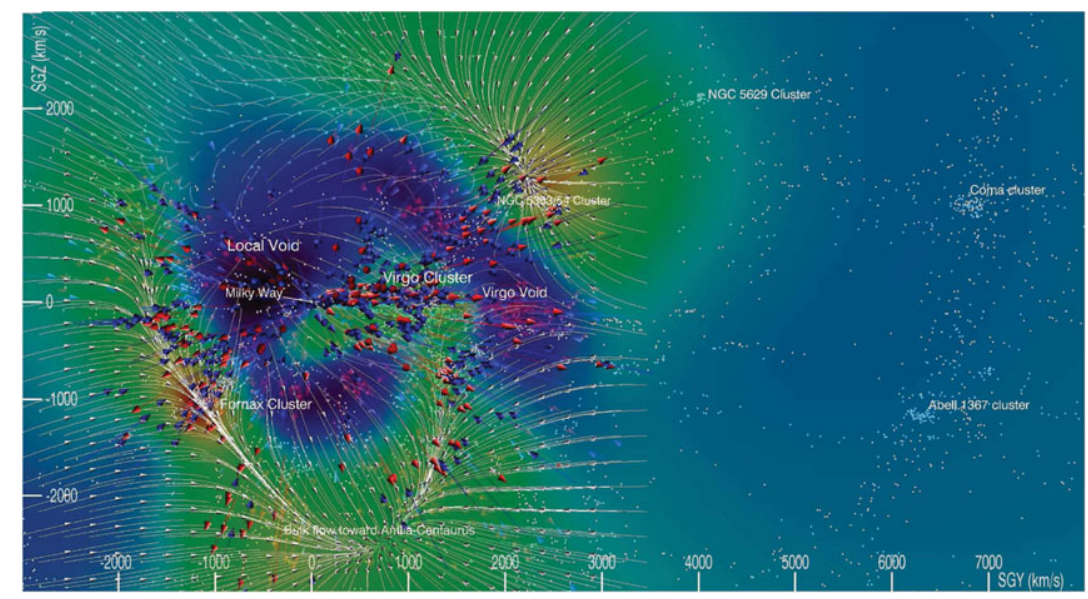

Figure 4. Visualization of structures and cosmic flows in the $S G X=0$ plane.

\section{Conclusions and perspectives}

The comparative visualization of 3D structures and flows enriches the study of the cosmography of the local Universe. A more detailed discussion will be presented in $\mathrm{H}$. Courtois et al. (in prep.). New observations that are currently being aggregated into the Cosmicflows-2 Catalog will provide distances of up to $10,000 \mathrm{~km} \mathrm{~s}^{-1}$. Reconstructions will extend our vision of cosmography to even larger scales.

\section{References}

Courtois, H. M., Hoffman, Y., Tully, R. B., \& Gottlöber, S. 2012, ApJ, 744, 43

de Vaucouleurs, G. 1956, Vistas in Astronomy, 2, 1584

de Vaucouleurs, G., de Vaucouleurs, A., Corwin, H. G., Jr., Butta, R. J., Paturel, G., \& Fouqué, P. 1991, Third Reference Catalogue of Bright Galaxies

Tully, R. B., Rizzi, L., Shaya, E. J., Courtois, H. M., Makarov, D. I., \& Jacobs, B. A. 2009, AJ, 138,323

Tully, R. B., Shaya, E. J., Karachentsev, I. D., Courtois, H. M., Kocevski, D. D., Rizzi, L., \& Peel, A. 2008, ApJ, 676, 184 